\title{
IMPACTO DE LA APLICACIÓN DE UNA GUIA METODOLOGICA CIENTIFICA EN PROYECTOS DE INVESTIGACIÓN ESCOLARES EN LA REGIÓN DE ATACAMA, CHILE
}

\author{
B. SEPULVEDA ${ }^{1}$, J. CASTRO ${ }^{2}$, O. PAVEZ ${ }^{3}$ \\ ${ }^{1,3}$ CRIDESAT-Universidad de Atacama, ${ }^{2}$ SEREMI de Educación de Atacama, Ministerio de Educación / Chile \\ bernardo.sepulveda@uda.cl ${ }^{1}$ \\ Recepción 23/11/2017 - Aprobación 24/01/2018 \\ DOI: $10.15628 /$ holos.2018.6595
}

\section{RESUMEN}

El aprendizaje de ciencias es, actualmente, un factor de desarrollo de los países. Proyectos estudiantiles de Copiapó (Atacama, Chile) presentados a concursos científicos nacionales, mostraron problemas de enfoque técnico y redacción inadecuada; posibles causas de aprobación de baja cantidad de proyectos. Además, no había una guía metodológica objetiva para que profesores y alumnos elaboraran proyectos. Se redactó una guía metodológica para transformar una idea común en idea de proyecto, herramienta de apoyo basada en un documento para alumnos de doctorado; orientada a estudiantes de colegio y con similar profundidad conceptual. En talleres en Atacama, a estudiantes y profesores se les aplicó la guía para estructurar la idea de investigación; asesorando la presentación y desarrollo de proyectos escolares, previo a cualquier formulario de proyectos. Correlacionado con lo anterior, se logró un premio como el Intel International Science and Engineering Fair en 2012 y 2013 y el aumento de participación exitosa en concursos. Se postula que la aplicación de esta guía permitió mejorar en calidad la presentación de proyectos y aumentar la aprobación.

PALABRAS CLAVE: Guía metodológica, proyectos escolares, idea de investigación, Región de Atacama.

\section{IMPACT OF THE APPLICATION OF A METHODOLOGICAL SCIENTIFIC GUIDE IN SCHOLAR RESEARCH PROJECTS IN THE ATACAMA REGION, CHILE}

\begin{abstract}
Science learning is currently a factor in the development of countries. In Copiapó (Atacama, Chile), school projects submitted to scientific national competitions showed problems due to lack of technical focusing and redaction; possible causes of admission of few projects. Moreover, there was not a methodological guide for teachers and students develop projects. A methodological guide to transform a common idea into a project idea was drafted. This support tool was based on a document made for doctoral students; oriented to college students and with similar conceptual depth. In workshops held in Atacama, the guide was applied to
\end{abstract}

students and teachers to structure an idea of research; advising the presentation and development of school projects, prior to any project form, and advising the presentation and development of student projects. Correlating with the above, between 2012 and 2013 an award was obtained as the Intel International Science and Engineering Fair and the increase of successful participation in competitions. It is postulated that the application of this guide allowed to improve in quality the presentation of projects and increase their approval.

KEYWORDS: Metodological guide, scholar projects, research idea, Región de Atacama. 


\section{INTRODUCCION}

El aprendizaje de la ciencia parece, hasta nuestro tiempo, ser un desafío particular para el desarrollo de los países. La educación promueve movilidad social e influye en el destino de los individuos (González, Martínez, Martínez, Cuevas y Muñoz, 2009); pero, se estima que el nivel de cultura familiar potencia la posibilidad de adquirir habilidades científicas (Zorlu et al., 2013) y que lo importante es la construcción del conocimiento como resultado de colaboración interdisciplinaria entre procesos individuales y sociales (Sullivan, 1998).

Actualmente los educadores están en posición para implementar nuevos sistemas de aprendizaje que brinden ventajas (Harris, Bransford y Brophy, 2002). Por ejemplo, el aprendizaje basado en problemas se informa más efectivo que la enseñanza tradicional en el desarrollo conceptual (Kartal y Bakaç, 2010). El concepto de enseñanza tradicional generalmente no se define con exactitud; si éste se refiere a la presentación de conocimiento en el aula centrada en el experto y sin desarrollo experiencial, la afirmación de los autores mencionados tendría importante consecuencia en el aprendizaje de la ciencia. Esto es porque la comprensión de la ciencia es vivencial; no puede estrictamente enseñarse, pues la experiencia en si debe adquirirse. Así, se explica la experiencia de estudiantes sometidos a aprendizaje ambiental fuera del aula, quienes opinaron que la ciencia es necesaria para comprender la vida; posteriormente la cantidad de estudiantes deseando ser científicos aumentó (Tasemir y Kartaly, 2012). El aprendizaje sería una experiencia emocional y cognitiva, el interés en aprender es afectado por el nivel de motivación; ello condujo a estudiantes a concentrarse en fuentes cognitivas, con la intención de continuar la práctica del sistema predicción - observación - explicación (Jon-Chiao, Ming-Yueh, Ming-Chou, Huei-Yin y YiLing, 2014). Así, el rol de la cultura podría impactar en estudiantes en la comprensión de la naturaleza del conocimiento, en la motivación para el aprendizaje de ciencia y en las relaciones interpersonales (Tzung-Jin, Feng, Ching-Sing y Chin-Chung, 2013). En análisis similar se resalta la importancia del nivel de auto eficacia para este aprendizaje (Ching-Chung, Hisin, Jyh-Chong y HungMing, 2011).

La educación en ciencia implica que el foco de un plan educativo debe centrarse en las diferentes capacidades de los educandos. Así, debe dársele importancia a adquirir habilidades en procesos científicos en la formación de profesores y que la adquisición de estas habilidades debe comenzar en la edad preescolar (Erkol y Ugul, 2014). Integrando cualidades necesarias para comprender la ciencia, Gilles et al. (2014) dicen que cuando los estudiantes aprenden cómo preguntar; entonces, inducen preguntas que los ayudan a investigar y explorar interrelaciones, generar hipótesis e inferencias, a ser cooperativos en actividades científicas y sostener discusiones sustanciales. Estas son habilidades críticas para el aprendizaje y comprensión de la ciencia (Gilles, Nichols, Burgh y Haynes, 2014).

En Chile, hay que mejorar el acceso al conocimiento y cultura científicos, para habilitar a la ciudadanía en la toma de decisiones (Macedo, 2008). En general, en el modelo educativo chileno, la metodología para formación es en ciencias y no en ciencia (la diferencia es muy importante) y se enfoca principalmente con la metodología común de las asignaturas. El principal síntoma observado fue un bajo desarrollo metodológico científico, de pensamiento crítico y redacción en estudiantes de diferentes niveles en Atacama. 
Por lo anterior, el objetivo de este trabajo fue aplicar una metodología para el aprendizaje de ciencia; en base a la construcción de la idea de proyecto de investigación para determinar una correlación con el mejoramiento en la presentación de proyectos de investigación científica a nivel escolar en la Región de Atacama. Se aplicó a estudiantes de educación básica y media de establecimientos educacionales de la Tercera Región; impulsando y asesorando el desarrollo y presentación de trabajos de carácter científico por estudiantes y asesorando a los profesores guía. Posteriormente, se evaluó resultados regionales en el mismo periodo.

\section{METOdOLOGÍA}

\subsection{Evaluación de calidad de proyectos de investigación estudiantil}

Se conformó una mesa técnica con profesionales experimentados en evaluación de proyectos científicos elaborados en establecimientos educacionales de la Región de Atacama, con fines competitivos y demostrativos (para instancias locales y nacionales). Se analizó la calidad de los proyectos en términos de redacción de objetivos y coherencia entre objetivo general y específicos, coherencia entre metodología y objetivos específicos, marco teórico, entre otros.

\subsection{Instrumento metodológico}

El resultado del análisis de la mesa técnica se usó para diseñar un instrumento de libre acceso para estudiantes y profesores. Los autores de este trabajo (CRIDESAT-UDA y SEREMI de Educación de Atacama) adaptaron una guía metodológica desarrollada para cursos de postgrado por el Dr. Bernardo Sepúlveda H., para producir un guion metodológico. Se redactó cada etapa de la redacción de un proyecto en forma amigable para todo usuario, cuidando de no especificar sesgos relacionados con profesiones, áreas de trabajo, títulos ni niveles de instrucción específicos e independiente de la edad. Se redactó la guía metodología para que de la redacción de una etapa se pueda obtener la siguiente, mediante una vinculación conceptual; reforzando la idea de que las secciones de un proyecto no son independientes sino interdependientes, lo que fue llamado internamente "estructura de persiana", debido a que un nivel cuelga dependientemente del anterior. Se reforzó la idea práctica de generar primero el objetivo general, como eje del proyecto y del cual se deduce los objetivos específicos. Lo anterior (objetivo general y específicos) es el núcleo del proyecto, por tanto las otras secciones se vincularon metodológica y directamente a él.

Esta guía metodológica no es un método para completar formularios, sino un esquema con lógica prediseñada para producir coordinadamente entre si las partes de una idea de proyecto desde una observación simple. Naturalmente que al obtener cada parte de la idea de proyecto y ubicarla correspondientemente en la sección de un formulario formal, se facilita el llenado de un formulario. Sin embargo son diferentes tareas y no siempre deben coincidir plenamente. Esta propuesta es una intelectualización autoguiada, lo que se manifiesta al comenzar lo formal con el objetivo general y no con el título como suele suceder cuando se confunde escribir la idea de un proyecto con rellenar un formulario de proyecto.

Se postuló que la aplicación de este instrumento podría mejorar la comprensión de la secuencia intelectual que representa la expresión de una idea redactada como proyecto, separando la gestación de la idea de proyecto de un formato de postulación. Por otra parte, se consideró que la 
aplicación del orden planteado en esta herramienta podría ayudar a acreditar las ideas como investigación que calcen en los formatos de corrección formal y a mostrar fundamentos metodológicos adecuados.

\subsection{Capacitación y talleres itinerantes}

Creada la guía metodológica, el programa de instrucción se ejecutó en talleres itinerantes titulados Talleres de transferencia metodológica para la investigación científica escolar. Se trabajó con 14 escuelas y 17 liceos de la región de Atacama. Participaron alumnos y profesores, no se impuso condiciones a los asistentes, salvo el interés de investigar. Los cursos se hicieron en las ciudades de Chañaral, Copiapó, Vallenar, Diego de Almagro y Huasco. Los talleres se iniciaron masivamente en julio de 2011 y se continuaron el año 2013 como asesoría y capacitación a grupos pequeños a solicitud de los establecimientos educacionales y clubes científicos.

Se resaltó la aplicación del método científico, no solo a la ciencia, sino a toda actividad, para ordenar y solucionar problemáticas de cualquier índole. Por otra parte, se buscó que los asistentes identificaran fortalezas y oportunidades regionales y locales, para difundir y explotar la actividad científica en Atacama. En primera instancia los educandos debían deducir el sentido del paso de una etapa a la siguiente.

\subsection{Evaluación del impacto por la aplicación del instrumento metodológico}

Se levantó información sobre el desempeño histórico, a nivel nacional y regional, de resultados de proyectos científicos escolares en dos instancias competitivas destacadas y de carácter nacional, como el Congreso Nacional Explora y el Concurso Junior del Agua Chile. En paralelo, se diseñó una línea de tiempo, en la cual se registró (a) la capacitación en bases metodológicas de la investigación científica, (b) la realización de encuentros de estudiantes en eventos de difusión y estimulación de la actividad científica escolar y (c) la presentación de proyectos en concursos de ciencia escolar, incluyendo el registro de posibles premios o logros en los concursos.

\section{RESULTADOS}

\subsection{Logros antes de la aplicación del programa}

En relación a la identificación de instancias (extracurriculares), como el desarrollo de las habilidades del pensamiento científico, hay diversas oportunidades y/o programas de amplia promoción, donde los estudiantes participan de experiencias formativas, competitivas y demostrativas de habilidades de la investigación científica escolar. En esta lógica, destaca el Programa Explora, de la Comisión Nacional de Ciencia y Tecnología (CONICYT), instrumento que entre sus variadas líneas de acción integra la Semana de las Ciencias, Congresos (regional y nacional) de Ciencia y Tecnología, Clubes Científicos, entre otros. Asimismo, destaca el concurso Junior del Agua; organizado en Chile por la Dirección General de Aguas, iniciativa de la Fundación de Agua de Estocolmo (SWF) establecida en 1990. Otra actividad es la Feria Científica Nacional Juvenil, organizada por el Museo Nacional de Historia Natural e iniciativas de cobertura local y nacional, 
como el Kimlu Campamento Científico, programa nacional de fomento de la vocación y educación en habilidades científicas de jóvenes de la Fundación Ciencia Joven.

La International Science and Engineeringy Fair (Intel ISEF), la competencia más grande a nivel mundial donde estudiantes de alrededor de 70 países exhiben en USA sus trabajos científicos, ofrece cada año a Chile dos cupos definidos en el Congreso Nacional de Explora-CONICYT.

Uno de los datos importantes recolectados fue que en términos de cantidad de alumnos premiados en el Congreso Nacional Explora-CONICYT en el periodo 2002-2012, de 130 alumnos premiados 8 son de Atacama (6\%). Lidera el ranking la Región Metropolitana.

En la Tabla 1 se muestra los congresos en que Atacama obtuvo premios. El galardón máximo que entrega el Congreso Explora es el premio Intel ISEF que recayó en Atacama en el Congreso XII (2011) y XIII (2013).

Tabla 1. Antecedentes de premios otorgados a la Región de Atacama, en el Congreso Nacional Explora-Conicyt, periodo, 2002-2012.

\begin{tabular}{|c|c|c|c|c|}
\hline Congreso & Año & Premio & Título proyecto & Establecimiento \\
\hline VII & 2006 & $\begin{array}{l}\text { RECONOCIMIENTO } \\
\text { EXPLORA }\end{array}$ & Cultivo de andenes tecnificado & $\begin{array}{c}\text { Liceo Manuel } \\
\text { Magalhaes Medling }\end{array}$ \\
\hline IX & 2008 & $\begin{array}{c}\text { MEJOR STAND } \\
\text { ENSEÑANZA MEDIA }\end{array}$ & $\begin{array}{c}\text { En boca cerrada se suben las notas. } \\
\text { Determinación de la relación entre } \\
\text { el nivel de presión acústica y el } \\
\text { rendimiento escolar }\end{array}$ & $\begin{array}{c}\text { Colegio San Agustín de } \\
\text { Atacama }\end{array}$ \\
\hline XII & 2011 & $\begin{array}{l}\text { EXPLORA A LA } \\
\text { INNOVACIÓN ISEF }\end{array}$ & $\begin{array}{l}\text { Cosa de pelos: Conservando el } \\
\text { recurso agua }\end{array}$ & $\begin{array}{c}\text { Colegio San Agustín de } \\
\text { Atacama }\end{array}$ \\
\hline XIII & 2012 & $\begin{array}{l}\text { EXPLORA A LA } \\
\text { INNOVACIÓN ISEF }\end{array}$ & $\begin{array}{l}\text { Creando un dispositivo que } \\
\text { aproveche el flujo de aire de un } \\
\text { vehículo para generar electricidad }\end{array}$ & Liceo Japón, Huasco \\
\hline
\end{tabular}

En la Figura 1 se muestra el comportamiento histórico del nivel de participación estudiantil en el Congreso Nacional Explora Conicyt (periodo 2002-2012). La tendencia global de la participación estudiantil en este evento nacional muestra un inicio de alta convocatoria que decayó hacia 2005; pero, que se ha mantenido estable el resto del periodo. 


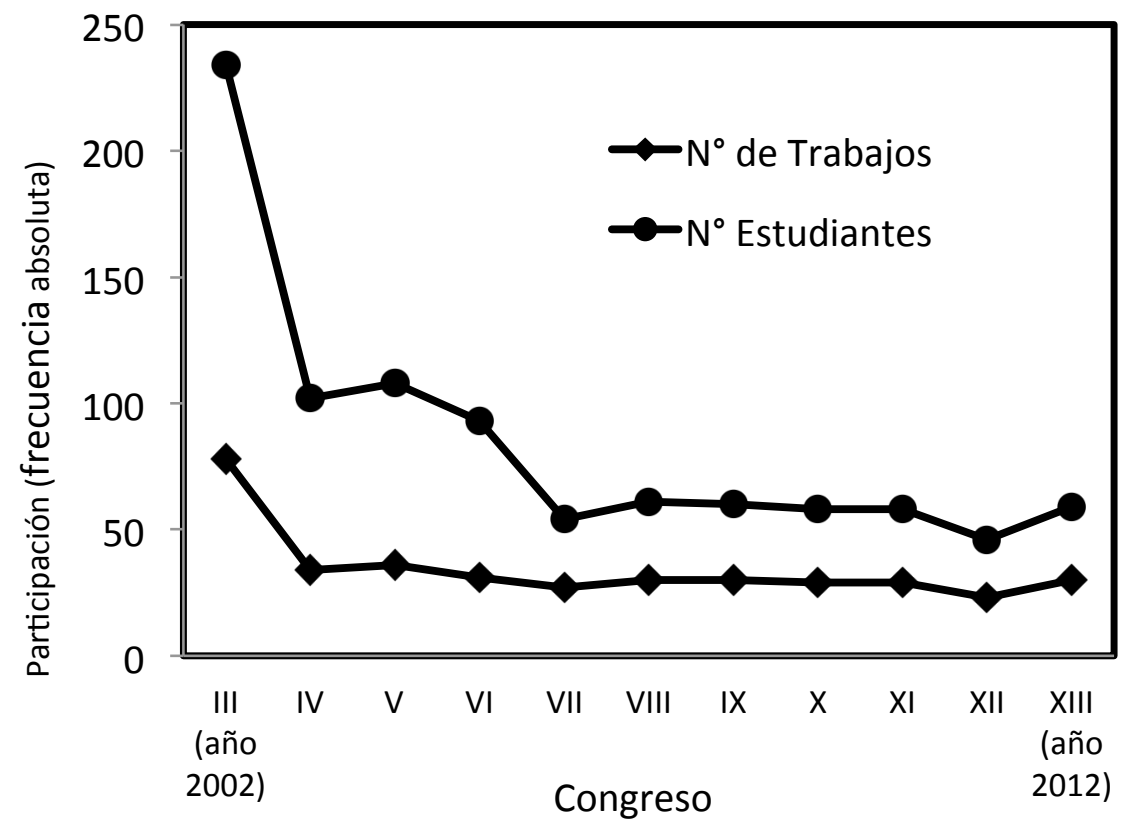

Figura 1. Comportamiento histórico del nivel de participación estudiantil en el Congreso Nacional Explora Conicyt (periodo 2002-2012). Fuente: Ministerio de Educación, Chile.

En la Tabla 2 se presenta el resultado histórico del concurso Junior del Agua Chile. En el periodo 2003-20013 en la región de Atacama se ha obtenido premios en los años 2003, 2004, 2011, 2012 y 2013. Al inicio de este concurso, Atacama exhibió un buen desempeño como fue el primer lugar año 2004, hasta el 2010 no se participó. Desde 2011 y hasta 2013 se ha logrado el segundo y tercer lugar nacional. Al concurso Junior del Agua se ha presentado un promedio de 12 proyectos, liderando la Región Metropolitana y la Zona Norte.

El instrumento metodológico generado para la realización de proyectos científicos, en contextos estudiantiles, se usó en los Talleres de transferencia metodológica para la investigación científica escolar y en paralelo se envió por oficio de la SEREMI de Educación a los establecimientos educacionales de la Región de Atacama.

\subsection{Evaluación de la Calidad en la Presentación de Proyectos}

En esta actividad se identificó problemas originales como (a) falta de coherencia entre método y objetivos y entre objetivos específicos y objetivo general; (b) incoherencia en la justificación con el tema a trabajar; (c) carencia de fundamentos bibliográficos o mal estructurado. En el diseño del instrumento, se tomó como principal motivo de los errores el que los usuarios no relacionan entre si las partes de un proyecto y si se hace, no se percibe el mecanismo que permite deducir la forma y el contenido de un ítem en relación a la idea central, que es el objetivo general y los objetivos específicos. Otro problema es la redacción y el contenido de cada ítem, se concluyó, por ejemplo, que hay una tendencia a mezclar objetivo(s) y metodología parcialmente, confundiendo el sentido del objetivo puro. Otro ejemplo, es que una de las unidades que produce problemas para realizarse es el fundamento teórico. Así, los problemas fueron la base para generar el guión metodológico. 
Tabla 2. Resultados históricos, concurso Junior del Agua Chile

\begin{tabular}{|c|c|c|}
\hline Proyecto & $\begin{array}{l}\text { Región/Premio: } \\
1,2,3)\end{array}$ & $\begin{array}{l}\text { Establecimiento } \\
\text { educacional }\end{array}$ \\
\hline $\begin{array}{l}\text { 2003: Tratamiento artesanal para reutilizar las aguas } \\
\text { empleadas en el proceso de cultivo del Ostión del Norte. } \\
\text { 2003: Eliminación de materia orgánica de los sedimentos } \\
\text { marinos utilizando la almeja Gari Sólida como una } \\
\text { herramienta de filtración. }\end{array}$ & Metrop ${ }^{1}$ & $\begin{array}{l}\text { L. Industrial José Tomás } \\
\text { de Urmeneta } \\
\text { Liceo García Manuel } \\
\text { Blanco Encalada }\end{array}$ \\
\hline $\begin{array}{l}\text { 2004: Control de la eutroficación de las aguas costeras con la } \\
\text { almeja gari sólida, "una herramienta de filtración natural". }\end{array}$ & $\| I^{1}$ & $\begin{array}{l}\text { Liceo Manuel Blanco } \\
\text { Encalada }\end{array}$ \\
\hline $\begin{array}{l}\text { 2005: Comparación de lugares costeros con diversas } \\
\text { actividades antropogénicas mediante la evaluación de sus } \\
\text { sedimentos con bioensayos de toxicidad. }\end{array}$ & VIIII & $\begin{array}{l}\text { Liceo Enrique Molina } \\
\text { Garmendia }\end{array}$ \\
\hline 2006: Cultivando el desierto con agua de mar. & $1^{1}$ & Liceo Elena Duvachelle \\
\hline $\begin{array}{l}\text { 2007: Presencia de plantas acuáticas vasculares en cuerpos } \\
\text { de agua dulce continental y su relación con el pH. }\end{array}$ & VIIII & Liceo Mauricio Hoschchild \\
\hline $\begin{array}{l}\text { 2008: Aislamiento y acción de bacterias descontaminantes } \\
\text { de petróleo. }\end{array}$ & Metrop ${ }^{1}$ & Instituto Luis Campino \\
\hline $\begin{array}{c}\text { 2009: Biorremediación de aguas contaminadas con boro } \\
\text { utilizando algas y la manipulación posterior de ellas para } \\
\text { producir fertilizante agrícola. }\end{array}$ & Metrop ${ }^{1}$ & Instituto Luis Campino \\
\hline 2010: Un Biosistema Integrado: Acuiponía. & $X 1^{1}$ & $\begin{array}{l}\text { Santa Teresa De Los } \\
\text { Andes }\end{array}$ \\
\hline 2011: Purificación del agua a través del cobre. & Metrop ${ }^{1}$ & $\begin{array}{l}\text { Fund. Educ. Colegio Ntra } \\
\text { Sra. de Lourdes. }\end{array}$ \\
\hline $\begin{array}{l}\text { 2011: Desarrollo de un dispositivo eficiente y de bajo costo, } \\
\text { para el mejoramiento de la calidad del agua de uso humano } \\
\text { y domiciliario, en contextos rurales vulnerables. }\end{array}$ & $\| I^{2}$ & Liceo José Santos Ossa \\
\hline $\begin{array}{l}\text { 2012: Creación de biocombustible a partir de residuos no } \\
\text { aprovechables de la industria salmonera. }\end{array}$ & $X I^{1}$ & Instituto Don Bosco \\
\hline 2012: Cosa de pelos: conservando el recurso agua. & $\| I I^{2}$ & $\begin{array}{l}\text { Col. San Agustín de } \\
\text { Atacama }\end{array}$ \\
\hline $\begin{array}{l}\text { 2012: Dispositivo capaz de desinfectar agua solo ocupando } \\
\text { el sol y materiales no biodegradables. }\end{array}$ & $11 \mathrm{I}^{3}$ & Col. Ambrosio O'Higgins \\
\hline $\begin{array}{l}\text { 2013: Búsqueda de microrganismos psicrófilos antárticos } \\
\text { capaces de degradar fenantreno para uso en } \\
\text { biorremediación de suelos y aguas contaminadas con } \\
\text { hidrocarburos aromáticos policíclicos (HAPs). }\end{array}$ & Metrop ${ }^{1}$ & $\begin{array}{l}\text { Liceo №1 de Niñas } \\
\text { Javiera Carrera }\end{array}$ \\
\hline 2013: Cantando en la ducha, recuperando el agua. & $\| I I^{2}$ & $\begin{array}{l}\text { Col. San Agustín de } \\
\text { Atacama }\end{array}$ \\
\hline 2013: Desde el sol de todos, hasta el agua nuestra. & $11 \mathrm{I}^{3}$ & $\begin{array}{l}\text { Liceo Católico de } \\
\text { Atacama / San Agustín de } \\
\text { Atacama }\end{array}$ \\
\hline
\end{tabular}




\subsection{Instrumento metodológico para diseño de idea de investigación}

Los cursos para instrucción en el uso de la guía metodológica para aplicación del método científico comenzaron a principios del 2011 y aún sigue vigente, siendo aplicado tanto periódicamente como en la forma de asesorías solicitadas por interesados. Estas actividades suelen programarse en las dependencias de los colegios y la atención a grupos específicos se ha estado haciendo en la Universidad de Atacama, específicamente en el Centro Regional de Investigación y Desarrollo Sustentable de Atacama, de la Universidad de Atacama, en donde concurren grupos de jóvenes investigadores con sus profesores guía. Naturalmente que lo anterior ha reforzado, en algún grado, la vinculación entre profesionales y docentes de Educación Básica y Media.

Por lo anterior, la atención a grupos de investigación escolar, con la instrucción en la metodología científica mediante esta guía metodológica, se ha convertido en una práctica sistemática, aunque no necesariamente con una frecuencia regular; ya que depende de las temporadas de presentación de proyectos. En cuanto a beneficiarios directos registrados, a la fecha, se anota alrededor de 320 personas. Respecto a la participación de estudiantes a la convocatoria de encuentros científicos estudiantiles masivos, se observó un incremento notable en los dos últimos años, con aproximadamente 1300 personas.

\subsection{Evaluación del impacto por la aplicación del instrumento metodológico}

Se estableció un análisis comparativo exante y expost de la fecha de inicio de los talleres de capacitación y del acceso al instrumento metodológico. Esto permitió observar que, exante la aplicación de la estrategia, el nivel de desempeño regional en instancias competitivas y/o demostrativas para ciencia escolar fue de $21 \%$; pero, se fortaleció desde 2011, para llegar a 63,3\% de participación. Para el caso del Concurso Junior del Agua, desde 2011 la Región de Atacama ha presentado consecutivamente proyectos, todos han sido preseleccionados y en la fase final siempre se ha obtenido a lo menos el segundo lugar nacional (Tabla 2).

En el caso del desempeño en el Congreso Nacional Explora, para los eventos antes del año 2011, los resultados indican premios pero de categorías inferiores, en cambio a partir del 2011 desde la Región de Atacama se ha obtenido el máximo galardón (Tabla 1), dos premios Intel-Isef consecutivos en 2012 y 2013, situación que nunca se había dado en los más de 10 años que la región había presentado proyectos al Congreso Nacional (Explora-CONICYT) que define este premio; la Tabla 3 detalla las delegaciones chilenas participantes en este concurso.

La Región de Atacama ha mejorado hacia el último segmento del periodo en cantidad de alumnos involucrados en espacios de participación al alero de la ciencia escolar, como campamentos o ferias, además de los premios obtenidos a nivel nacional. Otro antecedente, de data más reciente, está dado por la participación de la región en la 43ạ Feria Científica Juvenil que organiza el Museo Nacional de Historia Natural, donde de cinco proyectos presentados por la región fueron seleccionados tres, los cuales en octubre de 2013 compitieron con otros 33 proyectos del resto del país. 
Tabla 3. Delegaciones Chilenas participantes en el Congreso Nacional (Explora-CONICYT)

\begin{tabular}{|c|c|c|c|c|}
\hline Premio & Expositor & Región & Proyecto & Establecimiento \\
\hline $\begin{array}{c}\text { 2013: Phoenix, } \\
\text { Arizona. Intel } \\
\text { International } \\
\text { Science and } \\
\text { Engineering Fair }\end{array}$ & $\begin{array}{l}\text { Renzo Lombardi } \\
\text { Jonathan Hervoso }\end{array}$ & $\begin{array}{c}\text { Arica y } \\
\text { Parinacota }\end{array}$ & $\begin{array}{l}\text { Creando un dispositivo que } \\
\text { aproveche el flujo de aire de } \\
\text { un vehículo para generar } \\
\text { electricidad. } \\
\text { Sistema hidráulico } \\
\text { mareomotriz. }\end{array}$ & $\begin{array}{l}\text { Liceo Japón, } \\
\text { Huasco. }\end{array}$ \\
\hline $\begin{array}{l}\text { 2013: Pittsburgh, } \\
\text { Pennsylvania,USA. } \\
\text { Premio Explora a } \\
\text { la Innovación. }\end{array}$ & $\begin{array}{l}\text { Nicolás Castillo } \\
\text { Henry Díaz } \\
\text { Jorge Gutiérrez } \\
\text { Javier Romanos } \\
\text { Mariana Larroulet }\end{array}$ & Los Ríos & $\begin{array}{l}\text { Cosa de pelos: Conservando } \\
\text { el recurso agua. } \\
\text { Parasites inside thorns: } \\
\text { Morphological and genetic } \\
\text { characterization of the crab } \\
\text { Pinnaxodes chilensis. }\end{array}$ & $\begin{array}{l}\text { Colegio San } \\
\text { Agustín de } \\
\text { Atacama. } \\
\text { Instituto } \\
\text { Inmaculada } \\
\text { Concepción. }\end{array}$ \\
\hline
\end{tabular}

\section{DISCUSIÓN}

La política educativa del Estado chileno abre oportunidades concretas para la investigación científica a nivel estudiantil, ya que el marco curricular vigente propone el desafío formativo del desarrollo del pensamiento crítico como una meta transversal del curriculum escolar y establece el punto de inicio a un proceso educativo en ciencia. Lo anterior facultaría a estudiantes para hacer proyectos en asignaturas de ciencias, mediante la competencia en el manejo de las etapas de la redacción y concepción de un proyecto. Si bien en la Región de Atacama hay antecedentes de participación en proyectos; persiste una brecha importante de mejoramiento, la cual hay que abordar desde las capacidades técnicas regionales. En el aprendizaje de la ciencia, la mejor opción es el trabajo discípulo-maestro en un problema común (Cobarrubias y Toro, 2002); pero, profesores de aula que no han accedido a la metodología científica, difícilmente podrán generar proyectos de ciencia escolar con una base metodológica sólida. El problema de la preparación de los docentes en ciencia es, luego, correspondiente con los problemas que mostraban los proyectos presentados por estudiantes de nivel básico y medio en Copiapó y que fundamentaron probar la aplicación de una guía metodológica para estructurar una idea como propuesta de investigación.

Los problemas principales en enseñanza de la ciencia se enfocan en tres puntos. El primero es que las clases de ciencia se hacen del modo tradicional, no desarrollándose, inherentemente, habilidades de indagación. Un segundo punto es tener estrategias adecuadas para esta materia y, finalmente, las posibilidades de formación adecuada de profesores (Cofré, Camacho, Galaz, Jiménez, Santibañez y Vergara, 2010). Sobre este punto, en el trabajo de estos autores se plantea, además, que uno de los pilares de la formación de profesores en Chile es la "formación disciplinar o científica"; pero, "teniendo menor importancia otros dominios" como la investigación, la filosofía de 
las ciencias y la formación práctica". Esta afirmación es una confusión de ideas y muestra que no está claro el concepto de ciencia manejado a nivel de educación; la investigación y su práctica es la ciencia misma, pueden separarse conceptualmente; pero, para fines pedagógicos y nunca para la integridad de la formación científica. Los ejemplos temáticos y resultados no son la ciencia en sí y si lo es la metodología de razonamiento mediante el método científico, el cual es netamente práctico. Desde este punto de vista, los programas de formación de profesores de ciencias tienden a confundir el producto de las ciencias con la ciencia, cosa que se ha comprobado con frecuencia al analizar el desempeño de profesores de ciencias en el momento de producir una idea de proyecto, de ejecutarlo o de analizar el resultado (experiencia directa en programa Explora). En este aspecto, la dinámica llevada a cabo en este trabajo apunta, precisamente, a cubrir la falencia anterior; es decir, preparar a personas de todas las edades en la escencia de la ciencia, que es el pensamiento crítico y lógico. Aclarando un aspecto semántico, para que el pensamento crítico sea de nivel científico, por lo tanto objetivo, el sujeto debe educarse también mediante el método científico; es decir, potenciando el aprendizaje de la lógica para construir sistemas de comprobación de hipótesis y obtener respuestas bien fundamentadas que constituyan conocimiento científico. La adquisición de información en categoría de conocimiento científico implica que, antes de ejecutar cualquier trabajo, se debe planificar un diseño metodológico adecuado con una estructura autocomprobatoria de una hipótesis o propuesta, mediante contraste estadístico válido; es decir, sin olvidar el concepto de réplica. Este diseño metodológico refleja un modelo referencial. Por otra parte, el pensamento crítico puede existir en las personas y el aprendizaje del método científico puede contribuir como un mecanismo práctico para que las personas planteen y expliquen en forma simple y objetiva dicho pensamento crítico.

Los autores de este trabajo consideran, a la luz del resultado obtenido, que para la comunidad escolar el enfoque lógico de la metodología científica es desconocido y los alumnos prácticamente sienten que lo descubren. La mayor dificultad no ha sido la sencillez de la metodología científica, sino el que no es transmitida en colegios y universidades con la importancia que se le da a otras materias. Es decir, generar la actitud científica y el razonamiento crítico no tiene importancia por si mismo, sino que se considera componente obvio de materias específicas (biología, química, física, etc); lo que no es así. Lo anterior hace que la intención política de preparar a nuevas generaciones para el ámbito de la ciencia y la estrategia usada contrasten fuertemente. Este hecho quizá se deba a que la metodología científica sea considerada como un tema sofisticado de altura propia de grados académicos, o que solo se relaciona con temas de investigación de nivel puramente intelectual; pero, basicamente, no es correctamente comprendida. La metodología científica pudo llegar a personas de un amplio rango de instrucción (experiencias anterior B. Sepúlveda) y constituye una poderosa herramienta para buscar soluciones lógicas a las más diversas problemáticas, desde temas cotidianos hasta lo puramente científico. Así, en los distintos niveles de educandos en que se ha expuesto esta metodología, se detectó carencia en formación del pensamiento lógico. También, esta carencia se nota en el profesorado que debe guiar alumnos en asignaturas de ciencias desde la escuela primaria hasta la universidad. Del punto de vista semántico, a nivel de formación en ciencia hay, actualmente, una confusión entre enseñanza de ciencia y de ciencias; la ciencia en si es una unidad conceptual, es básicamente el método lógico y lo que se entrega a nivel docente es un compendio de resultados o contenidos generados en procesos científicos; pero, no la lógica metodológica que los genera. Naturalmente que el resultado de las ciencias es útil de conocer; pero, solo constituye información que no conduce al aprendizaje de la 
metodología. Los alumnos que se sometieron al curso llamado "ordenemos el pensamento" y los que, posteriormente, fueron asesorados en sus proyectos científicos, llegaron a concebir la diferencia; lo que se ha correlacionado con los resultados obtenidos.

En el proceso de aprendizaje de ciencia no se puede disociar los aspectos llamados teóricos de los prácticos. El concepto de ciencia implica en si el aspecto práctico o ejecutivo, el cual se debería manifestar en un cambio de actitud y juicio del educando al ver el mundo. La ciencia se refiere a una mentalidad analítica personal y el resultado práctico más inmediato es el desarrollo de la lógica y la crítica.

Merece especial atención que se desee preparar a ciudadanos en ciencias, mientras que no se destaca la formación en filosofia a nível de colegios desde nivel básico. El desarrollo del pensamiento razonable y crítico no se puede desvincular de la filosofía; ésta es vista, a nivel de educación media, más como una cuestión de historia del pensamento que como el desarrollo del razonamiento, casi sin conexión con la realidad diaria y muy alejada de la practicidad. La metodologia de la filosofia ha sido siempre una forma en que se ha solucionado grandes problemas de la humanidad; el pensamiento científico proviene de la filosofía.

El programa PISA de la OCDE señala que ser competente en el área de ciencias; "Implica no solo tener cierta información científica y la habilidad para manejarla, sino comprender también la naturaleza del conocimiento científico y de los poderes y limitaciones que dicho conocimiento tiene. Una formación científica completa debería, así mismo, fomentar en los estudiantes la convicción de que la ciencia puede modificar profundamente a la sociedad y a los individuos ..." (OCDE, 2006).

Un problema es que en el medio educativo coexisten visiones dogmáticas y constructivistas de la enseñanza y aprendizaje de las ciencias y competencias de pensamiento científico, independientemente del nivel de formación de los profesores de ciencia (Urra, 2011). Se ha tratado el tema de la educación mediante una serie de pasos, que incluye analizar los cambios que se están produciendo en la respectiva actualidad (Imbernón, Bartolomé, Flecha, Gimeno, Giroux, Macedo, McLaren, Popkewitz, Rigal, Subirats y Tortajada, 2010); estos cambios no deben verse sino como una transformación. En base a lo anterior, se debe determinar los retos para enfrentar la educación. Los mismos autores citan que la educación debe ser igualitaria, para dar un paso hacia una cultura educativa que convierta las dificultades en posibilidades $y$, finalmente, transformar las escuelas en comunidades de aprendizaje.

\section{CONCLUSIÓN}

Hacia el término del periodo $2010-2013$ se produjo un importante crecimiento en la presentación de proyectos de ciencia escolar en la Región de Atacama, respecto a cobertura y resultados en instancias competitivas y/o demostrativas.

Aunque el mejor resultado de la actividad científica escolar regional fue posterior a la instrucción en metodología, se podría elucubrar que la realización de las jornadas de instrucción con la guía de método científico y el actual apoyo de investigadores de la Universidad de Atacama y del Ministerio de Educación (MINEDUC, Copiapó, Chile), ha estimulado la aplicación de la guía del método científico como herramienta amigable y útil. Se postula que se ha contribuido a mejorar la comprensión de cómo estructurar una investigación. 
Se resalta la importancia y la necesidad del apoyo académico, así como la potencialidad de que el apoyo de la academia a la producción de ciencia escolar ayudaría a la potenciación del desarrollo regional.

En las sesiones de instrucción, se considera que se logró el entendimiento de que la comprensión y aplicación del método científico no depende, necesariamente, de la edad ni de la especialización en disciplinas, y que fue posible generar la actitud de investigación en jóvenes educandos. Esto es una oportunidad para aumentar el apoyo técnico de la academia y el MINEDUC, en la lógica de estimular la generación de un polo de desarrollo científico estudiantil local y/o regional.

\section{AGRADECIMIENTOS}

Los autores agradecen al Centro Regional de Investigación para el Desarrollo Sustentable de Atacama, de la Universidad de Atacama por el apoyo logístico y profesional en metodología en ciencias y a la Secretaría Regional Ministerial de Educación de Copiapó por apoyar la aplicación de la guía metodológica en establecimientos educacionales, en el plan de perfeccionamiento de la educación en la Región.

\section{REFERENCIAS}

Ching-Chung, T., Hisin, N.J., Jyh-Chong, L. y Hung-Ming, L. (2011). Scientific epistemic beliefs, conception of learning science and self-efficacy of learning science among high school students. Learning and Instruction, 21: 757-769.

Cofré, H., Camacho, J., Galaz, A., Jiménez, J., Santibañez, D. y Vergara, C. (2010). La educación científica en Chile: debilidades de la enseñanza y futuros desafíos de la educación de profesores. Estudios Pedagógicos XXXVI(2): 279-293.

Covarrubias, R.,y Toro, H. (2002). Ideas y planteamientos sobre la enseñanza de las ciencias biológicas. Revista chilena de Educación científica. 1(1): 7-21.

Erkol, S. e Ugul, I. (2014). Examining biology teachers candidates' scientific process skill levels and comparing these levels in terms of various varaibles. Procedia Social and Behavioural Sciences, 116: $4742-4747$.

Gilles, R.M., Nichols, K., Burgh, G. y Haynes, M. (2014). Primaty students' scientific reasoning and doscourse durin cooperativa inquiry-based science activities. International Journal of Education Development, 63: 127-140.

González, C., Martínez, M.T., Martínez, C., Cuevas, K. y Muñoz, L. (2009). La educación científica como apoyo a la movilidad social: desafíos en torno al rol del profesor secundario en la implementación de la indagación científica como enfoque pedagógico. Estudios Pedagógicos (Valdivia) 35(1):63-78.

Harris, T.R., Bransford, J.D. y Brophy, S.P. (2002). Roles for learning scienceand learning technologies in biomedical ingeeniering education: a review of recent advances. Annu. Rev. Eng., 4: 29-48. 
Invernon, F.(Coordinador), Bartolomé, L., Flecha, R., Gimeno, J., Giroux, H., Macedo, D., McLaren, P., Popkewitz, T.S., Rigal, L., Subirats, M. y Tortajada, I. (2010). La educación en el siglo XXI. Los retos del futuro inmediato. Ed. Graó (Barcelona), pp. 1-180.

Jon-Chao, H, Ming-Yueh, H., Ming-Chou, L., Huei-Yin, H. y Yi-Ling, Ch., (2014). Using a predictionobservation-explanation inquirí model to enhance student interest and intention to continue science learning predicted by their interconected cognitive failure. Computers \& Education, 72: 110-120.

Kartal, A. y Bakaç, M. (2010). The effect of problema based learning and traditional teaching methods on students'academic achievements, conceptual developments and scientific process skills according to their graduated high school types. Procedia Social and Behavioural Sciences, 2: 2409-2413.

Macedo, B. (2008). Cultura y formación científica: un derecho de todos. En: Didáctica de las ciencias, nueva perspectiva. $V$ Congreso internacional de Didáctica de las Ciencias. X Taller Internacional de Enseñanza de la Física. Barrios, C.S. (Compilador). Ministerio de Educación, Ed. Educación Cubana, La Habana, Cuba, pp.289. pp 22-28. ISBN 978-959-18-0350-4.

Organización para la Cooperación y el Desarrollo Económicos (OCDE) (2006). El programa PISA de la OCDE, qué es y para qué sirve. OCDE, USA, pp. 1-34.

Sullivan, A. (1998). Social constructivist perspectives on teaching and learning. Ann. Rev. Psychol., 49: 345-375.

Tasemir, A. y Kartaly, T. (2012). The use of out of the school learning environments forr the formation of scientific attitudes in teacher training programs. Procedio Social and Behavioural Sciences, 46: 2747-2752.

Tzung-Jin, L., Feng, D., Ching-Sing, Ch. y Chin-Chung, T. (2013). High school students'scientific epistemological beliefs, motivation in learning science, and their relationships: a comparative study within the chinese culture. International Journal of Education Development, 33: 37-47.

Urra, S.E. (2011). La noción de aprendizaje y enseñanza de las ciencias y su relación con la noción de competencias de pensamiento científico en profesorado de ciencias en formación. Universidad de Santiago de Chile, Facultad de Química y Biología, Departamento de Ciencias del Ambiente. Tesis de Pedagogía, pp. 1-66.

Zorlu, F. (2013). Examinating secondary school students' scientific process skillstermns of some variables. Procedio Social and Behavioural Sciences. 106: 1181-1189. 